\title{
FAULT RISK ASSESSMENT OF UNDERWATER VEHICLE STEERING SYSTEM BASED ON VIRTUAL PROTOTYPING AND MONTE CARLO SIMULATION
}

\author{
Deyu $\mathrm{He}^{1}$, Ph.D. \\ Niaoqing $\mathrm{Hu}^{1}$, Prof. \\ Lei Hu' ${ }^{1}$, Ph.D. \\ Ling Chen ${ }^{1}$, Ph.D. \\ Yiping Guo², Ph.D. \\ Shaoshan Chen ${ }^{3}$, Ph.D. \\ 1) National University of Defense Technology, Changsha, China \\ 2) China Shipbuilding Industry Corporation, Jiujiang, China \\ 3) Xichang Satellite Launch Center, Wenchang, China
}

\begin{abstract}
Assessing the risks of steering system faults in underwater vehicles is a human-machine-environment (HME) systematic safety field that studies faults in the steering system itself, the driver's human reliability (HR) and various environmental conditions. This paper proposed a fault risk assessment method for an underwater vehicle steering system based on virtual prototyping and Monte Carlo simulation. A virtual steering system prototype was established and validated to rectify a lack of historic fault data. Fault injection and simulation were conducted to acquire fault simulation data. A Monte Carlo simulation was adopted that integrated randomness due to the human operator and environment. Randomness and uncertainty of the human, machine and environment were integrated in the method to obtain a probabilistic risk indicator. To verify the proposed method, a case of stuck rudder fault (SRF) risk assessment was studied. This method may provide a novel solution for fault risk assessment of a vehicle or other general HME system.
\end{abstract}

Keywords: fault risk assessment; underwater vehicle; virtual prototyping; Monte Carlo simulation; steering system; fault simulation

\section{INTRODUCTION}

The steering system is a vital subsystem that controls the track and attitude of space motion of underwater vehicles, which have a large scale and complicated constitution. Faults in the steering system may be located anywhere and are usually transient and sudden. Current industrial production has addressed condition monitoring and health management for steering systems; however, fault detection at an early stage remains difficult. Thus, a fault in the steering system is very likely to lead to a fatal accident. Collision is the most common form, which often leads to an inability to obtain fault data. The above items result in a lack of comprehensive and available historic data, which presents a substantial challenge to qualitative risk assessment [1]

However, a serious fault in the steering system may not always lead to a fatal accident. This is because the adverse effect caused by the fault can be recovered via effective emergency operations. For example, if a stuck rudder fault (SRF) of the stern rudder occurs in an underwater vehicle, collision with the seabed may be avoided if recovery operations are followed to maintain an acceptable depth or to emerge from the water safely. This involves a "manoeuvring limitation": under the circumstances that are beyond the manoeuvring limitation, a fatal accident is inevitable; however, under the circumstances that are within the manoeuvring limitation, a fatal accident can be avoided via effective recovery operations. Manoeuvring limitations are generally dictated according to the current motion and environmental parameters when the fault occurs, such as speed, fault parameter and depth.

However, one possibility cannot be overlooked: under circumstances of potential recovery, a fatal accident finally occurs due to a failure to follow correct operation procedures (human error). Although the human error probability of a well-trained operator is very small, it should not be overlooked. Notably, many traffic accidents have been proven to be caused by human error. According to Ref [2-3], the human factor is the most important factor leading to highway transportation accidents, in which human error contributes to a large proportion. Additionally, human error probability could be considerably increased under emergency circumstances, which warrants detailed study to obtain a comparison for this change. 
According to the above analysis, risk assessments of an underwater vehicle related to a steering system fault address a typical HME systematic safety problem. To obtain a solution to this problem, the interrelationships between the three elements (human, machine and environment) should be comprehensively considered in one framework instead of investigating them individually. In fault risk assessment, though a fault in the machine is the essential risk, environment factors as well as human error can substantially influence the safety of the HME system. However, the randomness of the three elements aggravates the complexity of the assessment process.

Above all, to assess the risk posed to the vehicle caused by a steering system fault, two difficulties are confronted: the scarcity of fault data and the puzzle of randomness.

\section{LITERATURE REVIEW}

Simulation technology has been rapidly developing in recent decades following advances computer science; simulations are now being applied to nearly all domains of modern society. High efficiency, low cost and infinite flexibility account for the huge popularity of simulation technology [4]. Simulationbased safety/risk assessment is an important research trend, and researchers have been developing it for application in many issues such as those related to fires, energy, structures, environment, health, foods, and the economy [5-11]; this diversity also demonstrates the strong adaptability of simulations. Monte Carlo simulation is the most widely used method [12-15], primarily due to its capability to address problems of randomness and uncertainty.

Many trials and positive simulation-based results in assessing risks have been used to vehicles in recent years. Ref [16] proposed an accident risk assessment in marine transportation based on a Markov Chain Monte Carlo simulation that can consider any accident or marine system and that does not need large-scale data collection. Ref [17] proposed a risk assessment method of aero engine failure based on Monte Carlo simulation that has been claimed to be very flexible. Ref [18] proposed an assessment approach for safety performance of road locations based on a combination of statistical analysis, numerical modelling, and micro simulation models, which can be used to estimate the number of conflicts and injury severity of a particular road location. Ref [19] proposed a probabilistic assessment method for vehicle safety under various driving conditions utilizing dynamic simulation models and the response surface method.

Most methodologies proposed in the above studies can be classified into two categories. One category is to utilize a simulation method (usually Monte Carlo) to estimate the frequency of accidents in a particular region and period. This methodology can only provide a global estimation of accident occurrence but is unable to consider fault details (or other reasons) that lead to accidents. Therefore, this approach cannot provide guidance for the design or use of the vehicle to reduce risk. The other category uses a simulation method to estimate the probability of a particular fault. The primary disadvantage of these methodologies is that they disregard environmental and human factors that may affect system safety. Thus, they need to be improved for application in an HME systemic safety problem.

In this paper, to address the first and second difficulties stated in section 1, respectively, virtual prototyping and Monte Carlo simulation are adopted, both of which are combined to form a novel simulation-based method for fault risk assessment in an underwater vehicle steering system. To verify the proposed method, a case study of SRF risk assessment of the underwater vehicle is presented afterwards in section 3 . A conclusion is made in the last section.

\section{CASE STUDY PROCESS}

The steering system is an important subsystem that controls the track and attitude of space motion of underwater vehicles, which is a typical double closed- loop mechanic- electronichydraulic hybrid control system. It is a pump-cylinder servo system consisting of an operating device, pump control unit, cylinder and feedback unit, etc. Steering system has a large scale and complicated constitution. The actual rudder angle is controlled to track the order angle given by the operator via two-stage feedback control. The detailed principle of the steering system is illustrated in Fig. 1 [20], in which measurement points $1 \# \sim 7 \#$ are going to be used in the validation process of virtual prototyping.

\section{VIRTUAL PROTOTYPING AND ITS VALIDATION}

Virtual prototyping is a digital model of the actual system, which is essentially a modelling and simulation (M\&S) technique. Common platforms of virtual prototyping are AMESim, ADAMS [21], Pro/E, etc. Amesim is chosen as the modelling platform for virtual prototyping, and the established model is shown in Fig. 2, where measurement points 1\# 7\# correspond to Fig. 1, respectively. In the modelling process, a good balance should be achieved between the accuracy of the components and the complexity of the system [22].

For use in fault risk assessment, the established virtual prototyping needs to be verified and validated to ensure credibility and acceptability. A series of validation experiments was designed and implemented. Parts of the actual system and both the experiment plant and sensors are illustrated in Fig. 3.

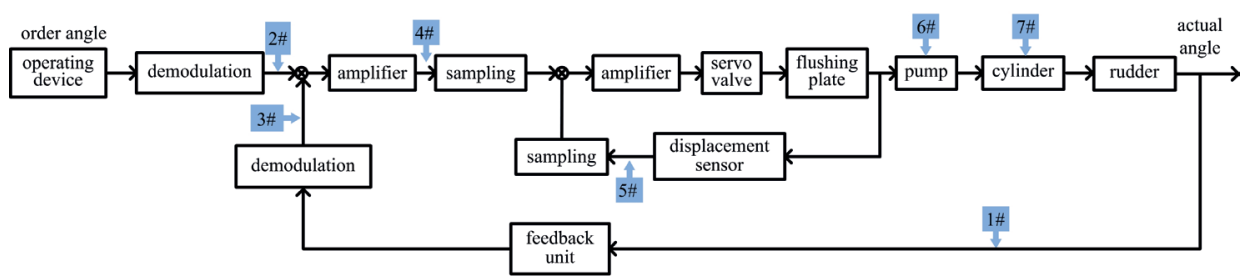

Fig. 1. Principle of steering system of underwater vehicle 


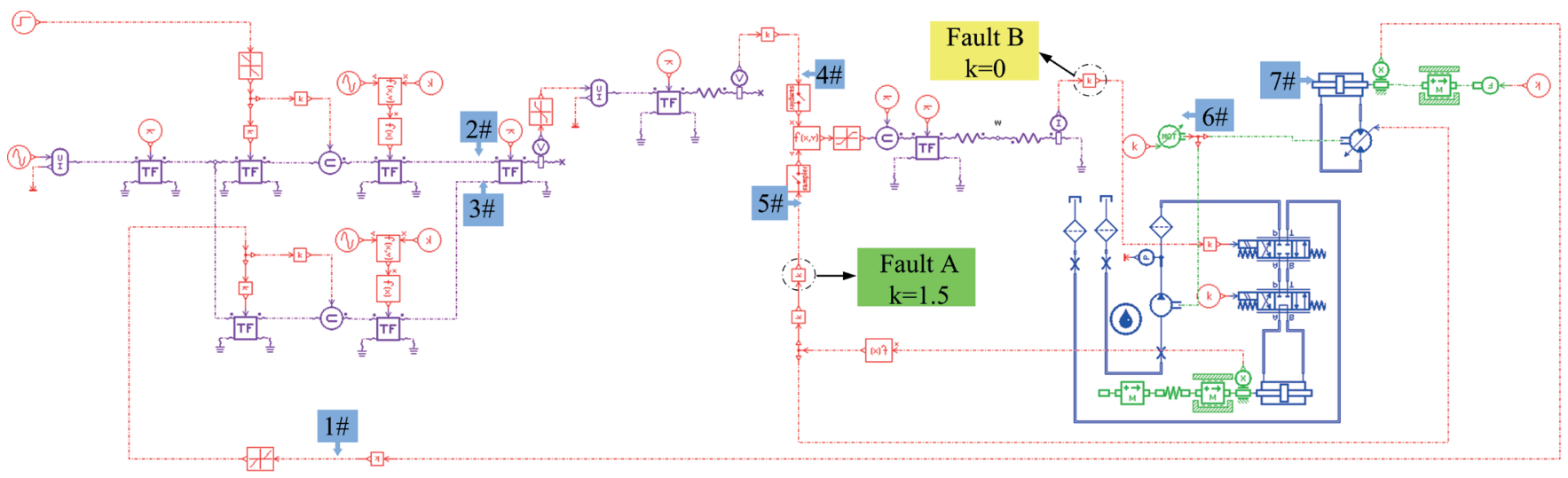

Fig. 2. Virtual prototyping of a steering system in Amesim. (Measurement points $1 \# \sim 7 \#$ correspond to those in Fig. 1. The components and parameters in green and yellow blocks are the fault injection details.)

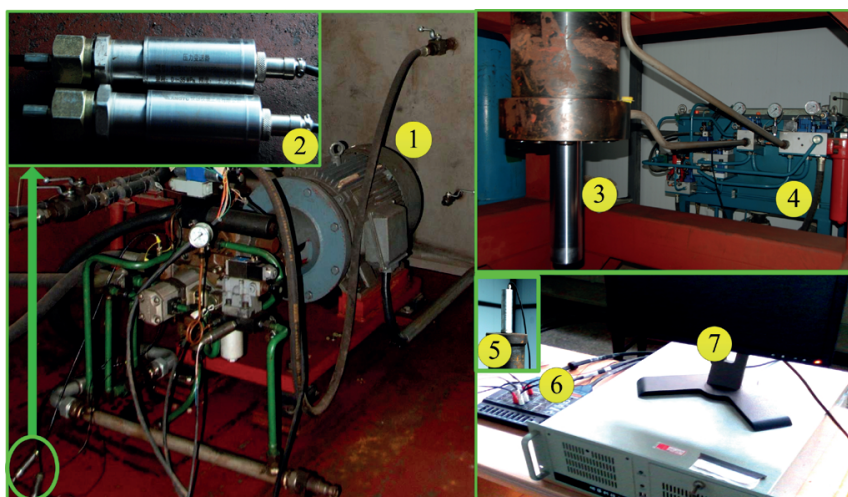

Fig. 3. Components of the actual steering system and experiment plant: 1 . pump control subsystem; 2. hydraulic pressure sensor; 3. cylinder; 4. loading plant; 5. displacement sensor of cylinder rod; 6 . data collector; 7. industrial computer

A detailed introduction and further discussion on verification, validation and accreditation (VV\&A) can be found in Ref [23][24][25]. This paper, however, only focuses on a comparison between the actual signal and a simulation signal of the measurement points. Suppose that $x_{t}$ and $y_{t}$ are the observation sequences of the actual system and virtual prototyping, respectively; the error sequence between them is

$$
e_{t}=x_{t}-y_{t}, t=1,2, \ldots, N
$$

where $\mathrm{N}$ is the length of the sequence.

The Root-Mean-Square (RMS) and Correlation Coefficient (CC) of the error sequence are chosen as the similarity indicators in $\mathrm{V} \& \mathrm{~V}$, which can reflect the similarity of the amplitude and the trend between the two sequences, respectively. Their calculation formulas are shown in (2) and (3).

$$
\mathrm{RMS}_{\mathrm{e}}=\sqrt{\frac{1}{N} \sum_{t=1}^{N} e_{t}^{2}}
$$

$$
\rho_{X, Y}=\frac{\sum_{t=1}^{N}\left(x_{t}-\bar{x}\right)\left(y_{t}-\bar{y}\right)}{\sqrt{\sum_{t=1}^{N}\left(x_{t}-\bar{x}\right)^{2} \sum_{t=1}^{N}\left(y_{t}-\bar{y}\right)^{2}}}
$$

The calculation results are shown in Tab. 1, and the comparison between the signals of the actual system and virtual prototyping of $5 \#$ and $7 \#$ are illustrated in Fig. 4. From Tab.1 and Fig. 5, it can be inferred that the actual signal and simulation signal have a high similarity, which indicates that the virtual prototyping has a high credibility and acceptability for the use of fault risk assessment.
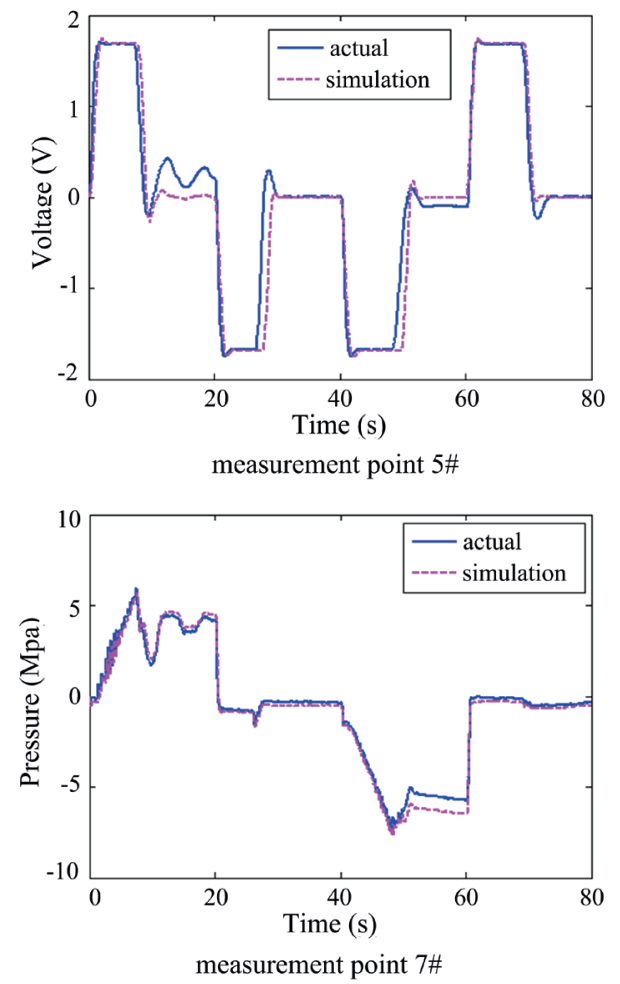

Fig. 4. Comparison between actual signal and simulation signal 
Tab. 1 Calculation result of similarity indicator

\begin{tabular}{|c|c|c|c|}
\hline No. & Signal & RMS $_{\mathrm{e}}$ & $\rho_{X, Y}$ \\
\hline $1 \#$ & Rudder angle $\left({ }^{\circ}\right)$ & 0.89 & 0.99 \\
\hline 2\# & Operation input (V) & 0.27 & 0.98 \\
\hline 3\# & Feedback input (V) & 0.07 & 0.99 \\
\hline 4\# & Amplifier output (V) & 0.52 & 0.96 \\
\hline 5\# & Displacement sensor (V) & 0.35 & 0.97 \\
\hline $6 \#$ & Pump difference (MPa) & 0.43 & 0.99 \\
\hline 7\# & Cylinder difference (MPa) & 0.49 & 0.99 \\
\hline
\end{tabular}

\section{FAULT INJECTION AND FAULT SIMULATION}

The steering system is a complicated mechanic-electronichydraulic hybrid control system. A fault in any component therein may lead to a failure in the entire system. There are nearly one hundred fault modes in the steering system according to its FMEA information, 25 of which are more typical and common. In this paper, two typical mechanical and electronic part failures, namely fault $A$ and fault $B$, respectively, indicate (A) the displacement sensor of the flushing plate failing to obtain a constant gain of 1.5 and (B) the proportional valve amplifier with a constant output signal of zero. These two faults are included in the virtual prototyping. According to the characteristics of the faults, appropriate library components are selected to imitate the faults, as illustrated in Fig. 2.

Fault simulation is then carried out on the basis of fault injection. The step signal is chosen as the order signal, the initial value of which is $30^{\circ}$. The value stepped to $0^{\circ}$ at $0 \mathrm{~s}$. The simulation period is $10 \mathrm{~s}$, and the sample interval is $0.1 \mathrm{~s}$. The simulation results of these two faults and their comparisons with normal virtual prototyping are illustrated in Fig. 5.

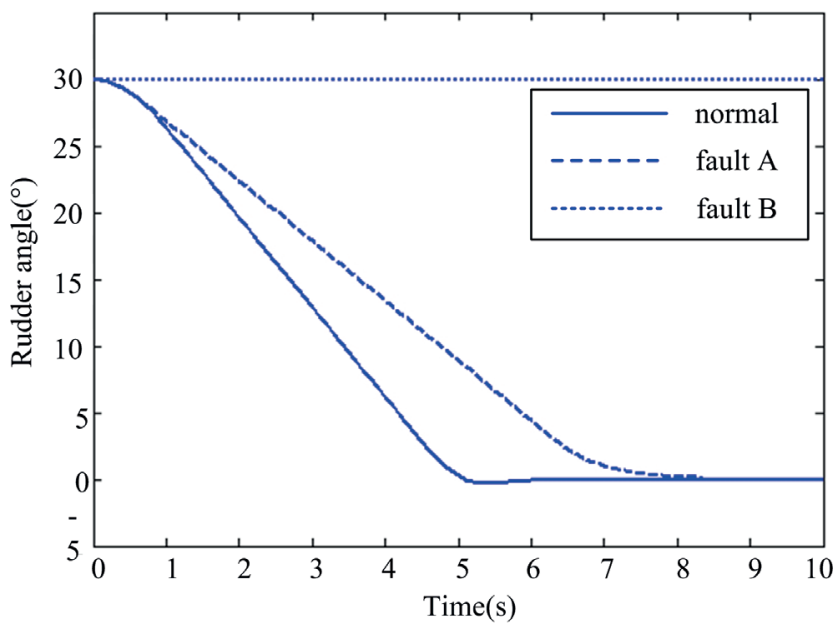

Fig. 5. Comparison between rudder angles of two faults with a normal condition

It can be inferred from the figure that fault B could lead to a stuck rudder and fault A could not, though it also appears to be a very serious fault. This is the reason and purpose for modelling virtual prototyping and conducting fault simulation: to determine which of the faults can lead to a stuck rudder. In other words, the effects of each fault mode are assessed regarding their effect on the rudder angle output in an attempt to determine whether a stuck rudder could precipitate. Similarly, the 23 other typical faults were integrated and simulated. This work may be very time-consuming. Based on the above works, all of the fault modes that can lead to a stuck rudder could be screened out in Tab. 2 , the failure rates (FR) of which are acquired from the design information, as listed in Tab. 2.

Tab. 2 Fault modes that can lead to SRF and their failure rates

\begin{tabular}{|c|c|c|}
\hline Component & Fault mode & Failure rate $\left(10^{-6} / \mathrm{h}\right)$ \\
\hline Operating unit & Transformer-constant- gain & 3 \\
\hline Demodulation 1 & Constant- output & 2 \\
\hline \multirow{2}{*}{ Pump control unit } & Proportional-valve-amplifier-zero-output & 65 \\
\hline & Proportional-valve-stops-working & 27 \\
\cline { 2 - 3 } & Pump failure & 25 \\
\hline Pump control power & Power circuit failure & 3 \\
\hline Cylinder & Cylinder failure & 0.01 \\
\hline Feedback unit & No-output & 2 \\
\hline Demodulation 2 & Constant- output & 2 \\
\hline Power unit & Constant- output & 1.5 \\
\hline & AC220 V V/400Hz power down & 1.5 \\
\hline
\end{tabular}

On this basis, a Reliability Block Diagram (RBD) is utilised to model the SRF probability of the steering system, as illustrated in Fig. 6.

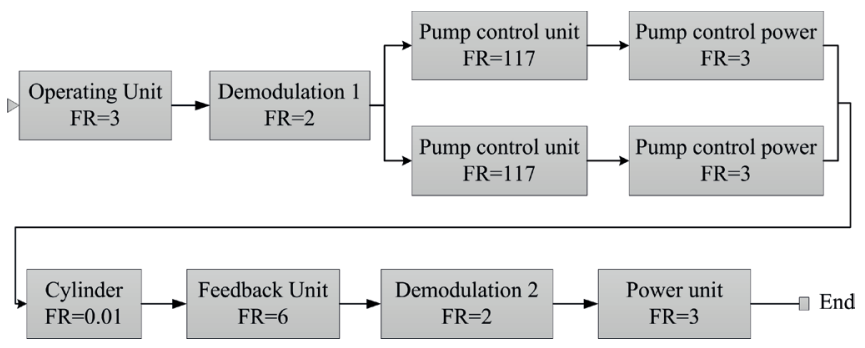

Fig. 6. RBD for SRF of steering system

From the RBD, SRF probability occurring at a particular time without considering maintenance can be acquired. If the vehicle is expected to constantly work for up to three months (2160 hours), the probability of SRF occurring at $2160 \mathrm{~h}$ is calculated at $3.46 \%$. Under this circumstance, the event of SRF occurring at $2160 \mathrm{~h}$ could be treated as a random event $\mathrm{X}$, which obeys a $0-1$ distribution. Suppose $\mathrm{X}=1$ when SRF occurs, then

$$
P\{X=k\}=0.0346^{k} \cdot 0.9654^{1-k}, k=0,1
$$

\section{MANOEUVRING LIMITATION DIAGRAM (MLD)}

MLD regulates the maximum submerging or emerging induced by the rudder angle as determined using speed, depth and trim. The MLD under a specified trim and sea depth consists of (1) a horisontal axis (speed U), (2) an ordinate axis (depth h), (3) a limitation curve of a stuck emerging induced by the stern rudder, and (4) a limitation curve of a stuck submerging induced by the stern rudder. A typical MLD of an underwater vehicle is illustrated in Fig. 7 (sea depth is $300 \mathrm{~m}$, trim is $\left.0^{\circ}\right)$. The region of "speed $\mathrm{U}(\mathrm{t})$-depth $\mathrm{h}(\mathrm{t})$ " could be divided into the following subregions [26]: 
(1) Safe region. In this subregion (with any speed or depth), although an SRF of submerging or emerging occurs when the stern rudder is at the maximum permitted angle, the vehicle could be recovered from colliding with the seabed or emerging from underwater via other available rudders (bow rudder and direction rudder) and reasonable emergency operations. The trim of the vehicle could be controlled within the permitted maximum trim $\theta_{\max }= \pm 30^{\circ}$.

(2) Emerging limitation region. In this subregion with a speed of $\mathrm{U}_{0}$, if an SRF of emerging occurs at the maximum permitted angle, the vehicle will inevitably rise to the surface or emerge from underwater.

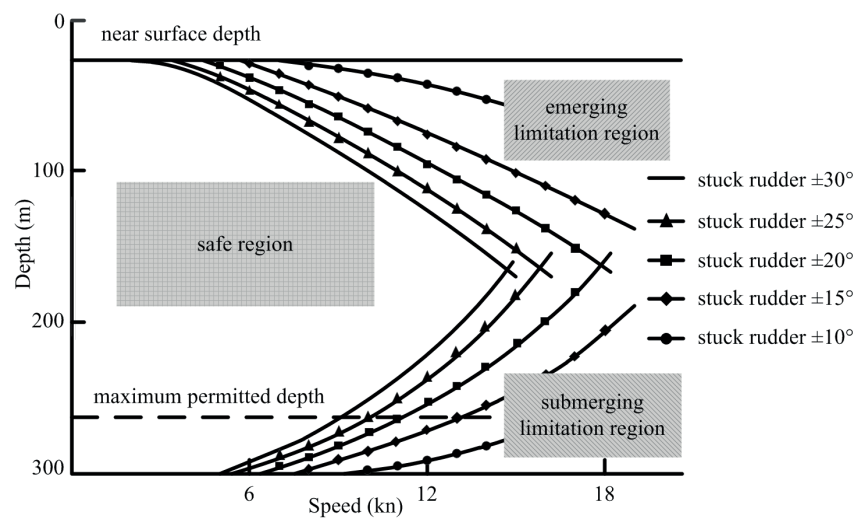

Fig. 7. A typical MLD (sea depth is $300 \mathrm{~m}$, trim is $0^{\circ}$ )

(3) Submerging limitation region. In this subregion with a speed of $U_{0}$, if an SRF of submerging occurs at the maximum permitted angle, the vehicle will inevitably descend toward or even collide with the seabed.

To obtain the MLD of an underwater vehicle, thousands of combinations of factors, such as the depth, speed, stuck rudder angle, trim and recovering operation, must be simulated and calculated. Though it is difficult to obtain the MLD, its detailed process is not a focus of this paper. However, the SRF of emerging is considered to be safe in this paper. That is to say, only the SRF of submerging could lead to risk (colliding with the seabed), which will be assessed in the following part.

\section{HUMAN RELIABILITY MODELLING}

When an SRF of submerging occurs in the steering system, various circumstances, such as depth and speed, may variously influence the operator. Obviously, if the current circumstance is very dangerous (close to the limitation), the operator may be so nervous that they may fail to conduct recovering operations to prevent a fatal accident. Thus, it is very necessary to model human error, which is another random event.

Firstly, to describe the severe level of the current circumstance when the fault occurs, a "fault threaten indicator" $\lambda €[0,1]$ is proposed in this paper. The larger the , the more dangerous the circumstance, which means that it is more adverse for carrying out recovering operations. Notably, $\lambda$ is determined by the current condition parameters when a fault occurs. An experience-based calculation formula of $\lambda$ is given as follows

$$
\begin{aligned}
\lambda & =\lambda_{e}+\left(\lambda_{\delta} \cdot \lambda_{h} \cdot \lambda_{U} \cdot \lambda_{\theta}\right)^{1 / 4} \\
& =\lambda_{e}+\left[\frac{1}{2} \frac{\delta_{\text {cur }}}{\delta_{\max }} \cdot \frac{h}{H} \cdot \frac{U_{\text {cur }}}{U_{\max }} \cdot\left(1-\frac{\theta_{\text {cur }}}{\theta_{\max }}\right)\right]^{1 / 4}
\end{aligned}
$$

Secondly, to describe the influence of on human error, a "mental influence indicator" $\eta €[0,1]$ is proposed in this paper. A larger indicates increased psychological pressure affecting the operator, thereby indicating an increased probability of human error. $\eta=1$ stands for a circumstance in which the psychological condition of the operator begins going against the recovering operations, which means the "mental stress" grade of the performance-shaping factors (PSFs) that influences the human reliability is only qualified to be 0.6 (Considering the strict selection and thorough training of the operator).

Suppose that $\lambda$ and $\eta$ have a relationship of an exponential positive correlation,

$$
\eta=\left(\mathrm{e}^{\lambda}-1\right) /(\mathrm{e}-1)
$$

Many techniques have been proposed for estimating human error probabilities (HEPs), including HEART (human error assessment and reduction technique), THERP (technique for human error rate prediction), and SLIM (success likelihood index methodology) [27]. Analytic hierarchy process (AHP) [28] is referred to in this paper. To simplify the research, detailed process of the method is not focused in this paper and HR is taken as a unary function of the mental stress grade. Other PSFs are assumed to be constant in the recovery process. Based on the analysis result of AHP and expert's experience, the weight of mental stress is supposed to be 0.15 and the human reliability in a normal condition is supposed to be 0.999 . Thus, the HR of a single operation considering the mental stress influence of fault threat is

$$
H R_{\mathrm{s}}=0.999-0.15 \times(1-0.6) \times \eta=0.999-0.06 \eta
$$

If SRF of submerging occurs, different recovering operation sets should be conducted according to the fault threat of the current circumstance, as listed in Tab. 3. Operation steps S1 to $\mathrm{S} 5$ are combined to form anti-sink manoeuvres with an increase in $\lambda$, each step of which obeys the human reliability formula (7).

Tab. 3 Anti-sink manoeuvres corresponding to different $\lambda$ and their total HR

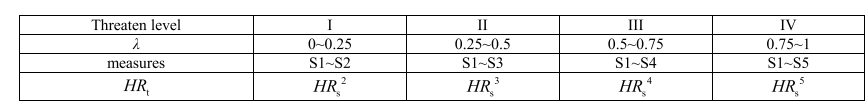

\section{RESULT AND DISCUSSION}

When the underwater vehicle is cruising in the sea, an SRF of submerging may occur under circumstances involving many random factors (PDFs of these are listed in Tab. 4). The PDFs of the first five factors are determined based on experience. Thus, these PDFs may vary according to the vehicle and working place. 


\begin{tabular}{|c|c|c|}
\hline Random factors & Distribution type & Distribution parameters \\
\hline Sea depth $H(\mathrm{~m})$ & Discrete uniform distribution & $H \sim U(50,300)$, interval=10 \\
\hline Diving depth $h(\mathrm{~m})$ & Continuous uniform distribution & $h \sim U(30, H)$ \\
\hline Trim $\theta\left(^{\circ}\right)$ & Discrete uniform distribution & $\theta \sim N(0,2)$, interval=1 \\
\hline Speed $U(\mathrm{kn})$ & Discrete uniform distribution & $U(3,24)$, interval=1 \\
\hline Stuck rudder angle $\delta\left(^{\circ}\right)$ & Discrete uniform distribution & $P(-30,30)$, interval=5 \\
\hline SRF & $(0-1)$ distribution & $k=0.0346^{k} \cdot 0.9654^{1-k}$, \\
\hline HR & $(0-1)$ distribution & $H R_{\mathrm{t}}=(0.999-0.06 \eta)^{n}$, \\
& & $n=2,3,4,5$ \\
\hline
\end{tabular}

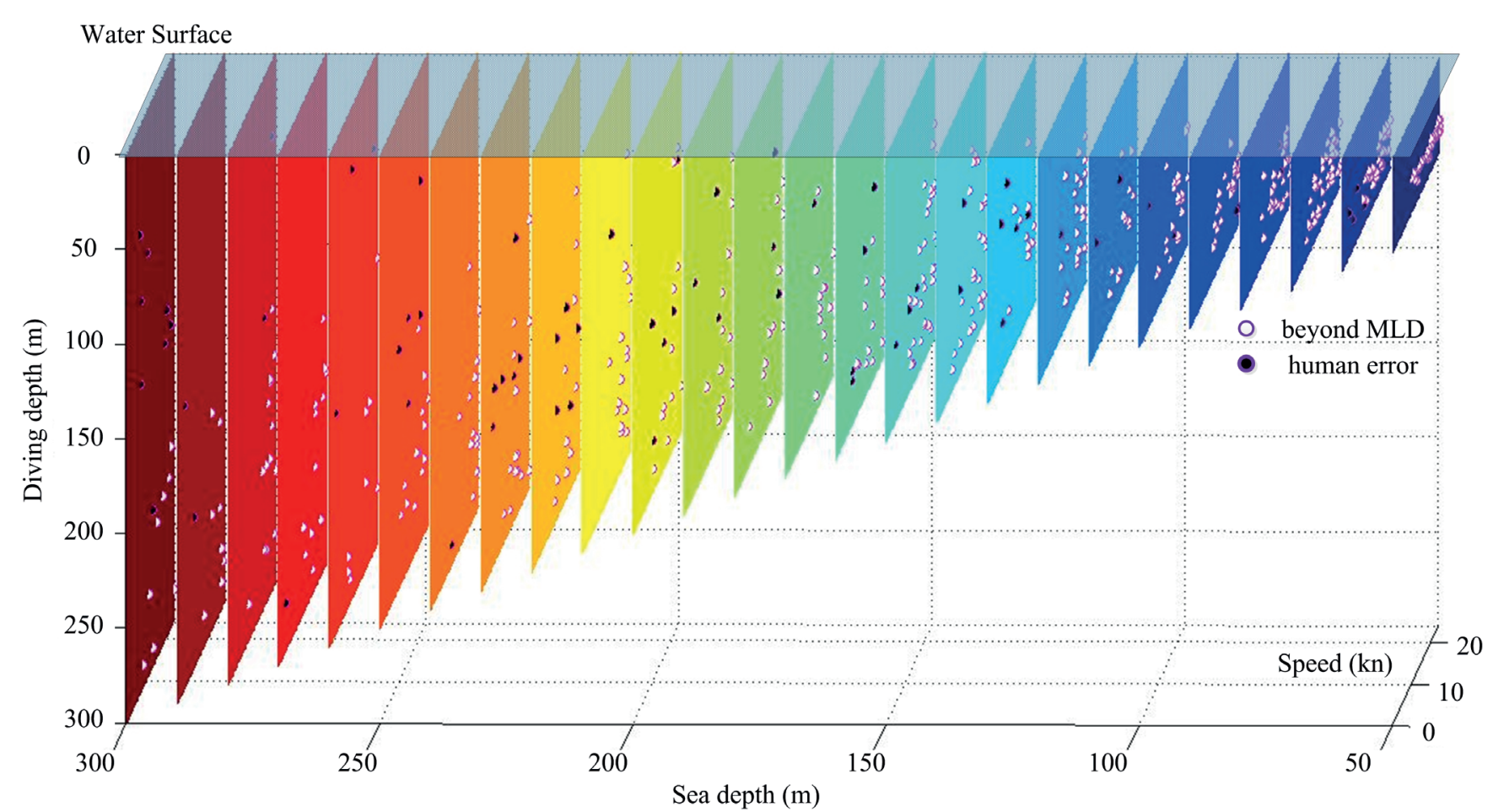

Fig. 8. Monte Carlo simulation result of fatal accident events (in $100 \mathrm{k}$ samples)

Based on the PDFs in Tab. 4 and MLD obtained in 3.3, a Monte Carlo simulation could be conducted. The volume of the sample is $100 \mathrm{k}$. In every simulation trial, a set of random values is used. In one simulation experiment, there are 479 events that are beyond the MLD; the number of events in which a fatal accident occurs due to human error within the MLD is 79, as illustrated in Fig. 8. Thus, the fatal risk (collision) probability of an SRF of the steering system is $(479+79) / 100000=0.558 \%$.

The distributions of all samples (left list) and fatal accidents (central list) as a function of stuck rudder angle, sea depth, diving depth, trim and speed are illustrated in Fig. 9. The right list of figures is the risk probability as a function of the five random factors, which is obtained based on the left and central list. The correlation coefficients of risk probability and each factor are also calculated.

The sensitivity of SRF risk to each factor can be inferred from Fig. 9. The distributions of all samples at each interval point are nearly the same for the stuck rudder angle and speed. However, the distributions of fatal accidents present an obvious rising trend, which means a remarkably positive correlation between the risk and the two factors. However, the situations are the opposite for the sea depth and trim. The decreasing trends, however, are not as notable as the above two. It is difficult to determine a distinct relationship between the diving depth and the risk. Therefore, the stuck rudder angle and the speed are the most vital factors in determining the risk of an SRF of the steering system of the underwater vehicle. It is recommended that the vehicle maintains a limited stern rudder angle and cruising speed, which is beneficial for successful recovery when SRF occurs.

\section{CONCLUSION}

In this paper, a fault risk assessment method for an underwater vehicle steering system based on virtual prototyping and Monte Carlo simulation is proposed; the method is then verified in a case study. Brief conclusions could be drawn as follows. 

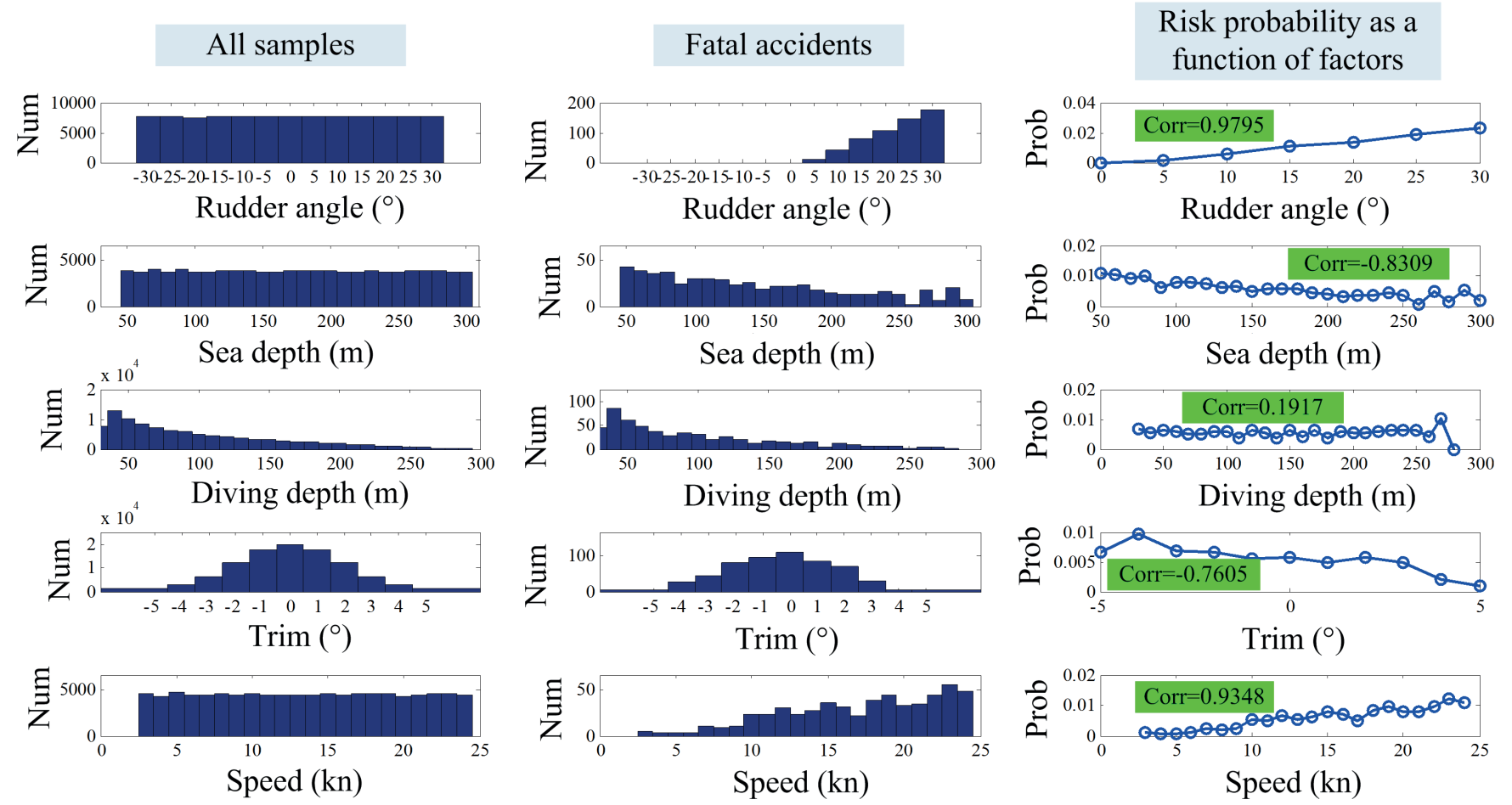

Risk probability as a function of factors

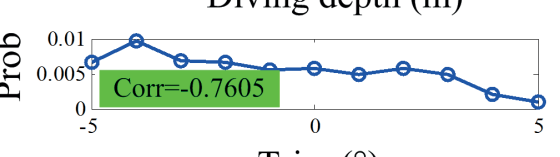

$\operatorname{Trim}\left({ }^{\circ}\right)$

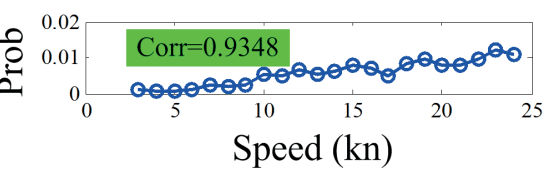

Fig. 9. Sensitivity analysis of the risk probability (100k samples)

(1) The establishment and V\&V of virtual prototyping is an important basis of the proposed method. The credibility of fault simulation data is directly determined using the similarity between the virtual prototyping and the actual system. Notably, instead of being demanded for all aspects, this similarity could be qualified for one particular use.

(2) Obtaining MLD is another important basis of the proposed method, which requires substantial calculation work. Different vehicles should have their own MLDs (except for extreme circumstances in which a fault leads to inevitable accidents); however, the nature of the MLDs may substantially differ.

(3) The distribution information of some important parameters of the vehicle is very important for fault risk assessment. In the case study of this paper, the PDFs of the five random factors are determined based on experience, which may not exactly fit reality. This may affect the accuracy of the risk assessment result. Therefore, the distribution information for random factors should be catalogued over time.

Overall, the method for fault risk assessment of an underwater vehicle steering system based on the combination of virtual prototyping and Monte Carlo simulation proposed in this paper fully incorporates the advantages of the simulation: high efficiency, low cost and infinite flexibility. The relationships between the three elements of the HME system and their influence on risk are comprehensively considered. This method provides a novel solution for fault risk assessment of vehicles and other general HMS systems. The primary shortcoming of this method is its large computation complexity. Further work should focus on efficiently obtaining MLDs for different vehicles and more accurate modelling of steering system faults.

\section{ACKNOWLEDGEMENTS}

This work was supported by the National Natural Science Foundation of China [grant number 51575518].

\section{BIBLIOGRAPHY}

1. Zhao Tingdi, Safety Design, Analysis and Validation. 2011, Beijing: National Defense Industry Press.

2. Qin Liyan, Shao Chunfu, and Jia Hongfei, Analysis on Express Way Traffic Accidents and Their Countermeasures. China Safety Science Journal, 2003. 13(6): p. 64-67.

3. LI Jin-long and S. Wan-hu, Cause Analysis ofTraffic Accidents on Express Highway and Study on Their Countermeasures. China Safety Science Journal, 2005. 15(1): p. 59-62.

4. Mei-Chen Hsueh, T.K.T., and Ravishankar K. Iyer, Fault Injection Techniques and Tools. Computer, 1997(April): p. 75-82.

5. Carmel, Y., et al., Assessing fire risk using Monte Carlo simulations of fire spread. Forest Ecology and Management, 2009. 257(1): p. 370-377.

6. Sarajcev, P., J. Vasilj, and R. Goic, Monte Carlo analysis of wind farm surge arresters risk of failure due to lightning surges. Renewable Energy, 2013. 57(0): p. 626-634. 
7. Rocha, J.M., A.A. Henriques, and R. Calçada, Probabilistic safety assessment of a short span high-speed railway bridge. Engineering Structures, 2014. 71(0): p. 99-111.

8. Olaru, M., M. Şandru, and I.C. Pirnea, Monte Carlo Method Application for Environmental Risks Impact Assessment in Investment Projects. Procedia - Social and Behavioral Sciences, 2014. 109(0): p. 940-943.

9. Lonati, G. and F. Zanoni, Monte-Carlo human health risk assessment of mercury emissions from a MSW gasification plant. Waste Management, 2013. 33(2): p. 347-355.

10. LeBlanc, D.I., et al., A national produce supply chain database for food safety risk analysis. Journal of Food Engineering, 2015. 147(0): p. 24-38.

11. Amigun, B., D. Petrie, and J. Görgens, Economic risk assessment of advanced process technologies for bioethanol production in South Africa: Monte Carlo analysis. Renewable Energy, 2011. 36(11): p. 3178-3186.

12. K. Durga Rao, V. Gopika, V.V.S. Sanyasi Rao, H.S. Kushwaha, A.K. Verma, A. Srividy, Dynamic fault tree analysis using Monte Carlo simulation in probabilistic safety assessment. Reliability Engineering and System Safety, 2009(94): p. 872-883.

13. Montewka, J., et al., A framework for risk assessment for maritime transportation systems - A case study for open sea collisions involving RoPax vessels. Reliability Engineering and System Safety, 2014(124): p. 142-157.

14. Ferrario, E. and E. Zio, Goal Tree Success Tree-Dynamic Master Logic Diagram and Monte Carlo simulation for the safety and resilience assessment of a multistate system of systems. Engineering Structures, 2014. 59(0): p. 411433.

15. Smid, J.H., et al., Strengths and weaknesses of Monte Carlo simulation models and Bayesian belief networks in microbial risk assessment. International Journal of Food Microbiology, 2010. 139, Supplement(0): p. S57-S63.

16. Faghih-Roohi, S., M. Xie, and K.M. Ng, Accident risk assessment in marine transportation via Markov modelling and Markov Chain Monte Carlo simulation. Ocean Engineering, 2014. 91(0): p. 363-370.

17. Chen, H., L. Li, and Y. Sun, Risk Assessment of Aero Engine Failure based on Monte Carlo Simulation. Procedia Engineering, 2014. 80(0): p. 415-423.

18. Sobhani, A., W. Young, and M. Sarvi, A simulation based approach to assess the safety performance of road locations. Transportation Research Part C: Emerging Technologies, 2013. 32(0): p. 144-158.
19. Chen, F. and S. Chen, Probabilistic Assessment of Vehicle Safety under Various Driving Conditions: A Reliability Approach. Procedia - Social and Behavioral Sciences, 2013. 96(0): p. 2414-2424.

20. HU Liang-mou, CAO Ke-qiang, and XU Hao-jun, Fault Diagnosis for Hydraulic Actuator Double Closed-loop System Based on Improved LS-SVM. Journal of System Simulation, 2009. 21(17): p. 5477-5480.

21. Zhe Cheng. A Hybrid Prognostics Approach to Estimate the Residual Useful Life of a Planetary Gearbox with a Local Defect, Journal of Vibroengineering, 2015, 17(2): 682-694.

22. Yang Chen, G.C., Zhenpeng Zhang, Yulong Huang, Multi-field coupling dynamic modeling and simulation of turbine test rig gas system. Simulation Modelling Practice and Theory, 2014(44): p. 95-118.

23. Balci, O., Verification, Validation and Accreditation, in Winter Simulation Conference. 1998. p. 41-48.

24. 5000.61, DoD, Verification Validation and Accreditation (VV\&A) Recommended Practice Guide, 1996.

25. MIL-STD-3022, DoD, Documentation of Verification, Validation, and Accreditation (VV\&A) for Models and Simulations 2008

26. WANG Jing-qi, SHI Sheng-da, and ZHANG Wei-kang, Safe Maneuvering Technology for Ensuring Submarine's Survivability. Journal of Naval University of Engineering, 2009. 21(1): p. 63-67.

27. Khan, F.I., P.R. Amyotte, and D.G. DiMattia, HEPI: A new tool for human error probability calculation for offshore operation. Safety Science, 2006. 44(4): p. 313-334.

28. Park, K.S. and J.i. Lee, A new method for estimating human error probabilities: AHP-SLIM. Reliability Engineering \& System Safety, 2008. 93(4): p. 578-587. 


\title{
CONTACT WITH THE AUTHORS
}

\author{
Deyu $\mathrm{He}$ \\ Niaoqing $\mathrm{Hu}$ \\ Lei $\mathrm{Hu}$ \\ Ling Chen \\ email: hedeyu@nudt.edu.cn \\ Laboratory of Science and Technology on Integrated \\ Logistics Support \\ National University of Defense Technology \\ Changsha 410073 \\ CHINA \\ YiPing Guo \\ email: 1696816984@qq.com \\ The Jiujiang Branch of 707 Research Institution \\ China Shipbuilding Industry Corporation \\ Jiujiang 332007 \\ CHINA \\ Shaoshan Chen \\ email: 492478230@qq.com \\ Xichang Satellite Launch Center \\ Wenchang 571300 \\ CHINA
}

